\title{
Zur Berechnung von mittleren Schwingungsamplituden
}

\author{
Normalkoordinatenanalyse und Ermittlung von mittleren Schwingungsamplituden \\ nach einer neuen Methode für Moleküle des Typs $\mathrm{XY}_{3}\left(\mathrm{C}_{3 \mathrm{v}}\right)$
}

\author{
A. Müller, B. Krebs und C. J. Peacock \\ Anorganisch-Chemisches Institut der Universität Göttingen \\ (Z. Naturforsch. 23 a, 1024-1028 [1968] ; eingegangen am 31. März 1968)
}

\begin{abstract}
Some rules for mean amplitudes of vibration $u$ are given. It is shown that the $u$ values for bonded atoms can be calculated by a simple formula needing only stretching force constants and atomic masses. The values obtained for a number of $\mathrm{XY}_{3}\left(\mathrm{C}_{3 \mathrm{v}}\right)$ and $\mathrm{XY}_{4}\left(\mathrm{~T}_{\mathrm{d}}\right)$ molecules show generally less than $5 \%$ difference from the rigorously calculated ones. The data published in the literature are often several times too large. For the $\mathrm{XY}_{3}\left(\mathrm{C}_{3 \mathrm{v}}\right)$ molecules a normal coordinate analysis in a M.V.F.F. approximation is given. From a new simple $\boldsymbol{L}$ matrix approximation method mean amplitudes of vibration have been calculated for these species which also agree very well with rigorously calculated ones.
\end{abstract}

Vergleicht man die von verschiedenen Autoren aus spektroskopischen Daten berechneten mittleren Schwingungsamplituden miteinander, so stellt man fest, daß sich die Werte für das gleiche Molekül zum Teil völlig voneinander unterscheiden *. In der folgenden Arbeit sollen einige Regeln über die Wertebereiche von mittleren Schwingungsamplituden angegeben werden und eine zuverlässige Abschätzungsmethode für die Amplituden bei gebundenen Atomen vorgeschlagen werden. Weiterhin soll eine Normalkoordinatenanalyse für Moleküle und Ionen des Typs $\mathrm{XY}_{3}\left(\mathrm{C}_{3 \mathrm{v}}\right)$ durchgeführt werden und für die gleichen Spezies mittlere Schwingungsamplituden nach verschiedenen Methoden berechnet werden, da die in der Literatur angegebenen Werte zum größten Teil falsch sind (vgl. ${ }^{\mathbf{1}}$ ).

\section{I. Über mittlere Schwingungsamplituden}

In der Literatur werden z. B. von Nagarajan und Radhakrishnan folgende mittlere Schwingungsamplituden angegeben (die Literatur hierzu ist dem Buch von Cyvin $^{1} \mathrm{zu}$ entnehmen): (alle Angaben für $298{ }^{\circ} \mathrm{K}$ )

$$
\begin{aligned}
& \mathrm{PBr}_{3}: \quad u_{\mathrm{P}-\mathrm{Br}}=0,0855 ; \quad \mathrm{PJ}_{3}: \quad u_{\mathrm{P}-\mathrm{J}}=0,0992 \text {; } \\
& \mathrm{XeO}_{3}: \quad u_{\mathrm{Xe}-0}=0,0663 \text {; } \\
& \mathrm{AlCl}_{4}{ }^{-}: \quad u_{\mathrm{AlCl}}=0,0759730 \text {; } \\
& \mathrm{AsCl}_{4}{ }^{+}: \quad u_{\mathrm{As}-\mathrm{Cl}}=0,0721937 \text {; } \\
& \mathrm{InCl}_{4}^{-}: \quad u_{\mathrm{In}-\mathrm{Cl}}=0,1595127 \AA \text {. }
\end{aligned}
$$

Die Werte sind zum Teil um einen Faktor 3 falsch.

* Sämtliche Arbeiten bis 1966 sind in dem ausgezeichneten Buch von Crvin ${ }^{1}$ zitiert.

1 S. J. Crvin, Mean Square Amplitudes and Molecular Vibrations, Universitetsforlaget, Oslo (im Druck).
Der Grund für die zahlreichen fehlerhaften Berechnungen liegt darin, daß die eingeführten Näherungen, z. B. die Vernachlässigung von Nichtdiagonalelementen der $\boldsymbol{\Sigma}$-Matrix bei der Lösung des Säkulargleichungsproblems ${ }^{1}$

$$
\operatorname{det}\left|\boldsymbol{\Sigma} \boldsymbol{G}^{-1}-\boldsymbol{\Delta} \boldsymbol{E}\right|=0
$$

in vielen Fällen unvernünftig sind. Bei starken Kopplungen können die $\Sigma_{i j}(i \neq j)$-Glieder von der gleichen Größenordnung sein wie die $\Sigma_{i i}$-Elemente ${ }^{2}$. In solchen Fällen versagt auch ein Näherungsverfahren von Torkington (vgl. ${ }^{2}$ ), das in zahlreichen Arbeiten von Nagarajan benutzt worden ist.

Hier soll nun eine Abschätzungsmethode zur Ermittlung von $u_{\mathrm{X}-\mathrm{Y}}$-Werten angegeben werden, die lediglich die Kenntnis der entsprechenden Kraftkonstanten voraussetzt $\left(f_{\mathrm{X}-\mathrm{Y}}\right)$ und im Rahmen der verwendeten Näherungen zuverlässig ist.

Für die mittlere Schwingungsamplitude eines gebundenen Abstandes gilt folgende Näherungsgleichung ${ }^{3}$ bei Schwingungsfrequenzen $v_{i}<1200 \mathrm{~cm}^{-1}$ und für $T \approx 300^{\circ} \mathrm{K}$ :

$$
u_{\mathrm{X}-\mathrm{Y}}^{2}=k T\left(F^{-1}\right)_{i i}+\frac{h^{2}}{64 \pi^{2} k T}\left(\mu_{\mathrm{X}}+\mu_{\mathrm{Y}}\right) .
$$

$\boldsymbol{F}^{-1}$ ist die reziproke $\boldsymbol{F}$-Matrix mit den Elementen $f_{i j}$ in inneren Koordinaten. Das $i i$-Element ist dasjenige, das der Koordinate $\mathrm{X}-\mathrm{Y}$ entspricht. Mit

2 A. Müller, Z. Phys. Chem. 238 [1968], im Druck.

3 Y. Morino, K. Kuchitsu, A. Takahashi u. K. Maeda, J. Chem. Phys. 21, 1927 [1953]. 
der Näherung (vgl. ${ }^{7,8}$ )

$$
\left(F^{-1}\right)_{i i} \approx f_{i i}{ }^{-1} \triangleq f_{\mathrm{X}-\mathrm{Y}^{-1}}
$$

erhält man aus (1) ${ }^{* *}$ :

$$
u_{\mathrm{X}-\mathrm{Y}}=\left[k T f_{\mathrm{X}-\mathrm{Y}}{ }^{-1}+\frac{h^{2}}{64 \pi^{2} k T}\left(\mu_{\mathrm{X}}+\mu_{\mathrm{Y}}\right)\right]^{1 / 2} \text {. }
$$

Die Näherung (2) ist sinnvoll, wenn die Nichtdiagonalelemente der $\boldsymbol{F}$-Matrix nicht zu groß sind; im Grenzfall völlig ungekoppelter Schwingungen gilt (2) exakt. Gleichung (3) ergibt anschaulich, daß mit zunehmender Valenzkraftkonstante und zunehmender Summe der reziproken Massen die mittleren Schwingungsamplituden zunehmen.

Wir haben nun nach (3) für eine Reihe von Molekülen und Ionen des Typs $\mathrm{XY}_{4}$ mit $\mathrm{T}_{\mathrm{d}}$-Symmetrie und des Typs $\mathrm{XY}_{3}$ mit $\mathrm{C}_{3 \mathrm{v}}$-Symmetrie $u$-Werte berechnet und mit denjenigen verglichen, die von MüLLER und Cyvin ${ }^{4}$ bzw. in dieser Arbeit nach bekannter Methode ${ }^{1}$ ermittelt worden sind. Die Valenzkraftkonstanten $f_{\mathrm{X}-\mathrm{Y}}$ sind für $\mathrm{XY}_{4}\left(\mathrm{~T}_{\mathrm{d}}\right)$ einer Arbeit von KREBS, MüLLER und $\mathrm{F}_{\text {ADINI }}{ }^{5}$ und für $\mathrm{XY}_{3}\left(\mathrm{C}_{3 \mathrm{v}}\right)$ dem Buch von Siebert ${ }^{6}$ entnommen. Die Tabellen 1 und 2 zeigen, daß sich in allen Fällen eine sehr gute Übereinstimmung ergibt. Die Abweichung der Näherungswerte ist jedoch um so größer, je größer die Kopplung zwischen den Schwingungen zur gleichen irreduziblen Darstellung ist, z. B. wenn $m_{\mathrm{Y}} / m_{\mathrm{X}}$ zunimmt.

$\mathrm{Da}$ sich mit (3) trotz der groben zugrunde gelegten Näherungen brauchbare $u$-Werte ergeben, liegt an der Unempfindlichkeit der $u$-Werte gegenüber dem Potentialmodell (vgl. ${ }^{2}$ ).

Aus Gl. (2) lassen sich nun folgende Schlüsse ziehen und Regeln ableiten:

1. Die mittlere Schwingungsamplitude für eine bestimmte Bindung $\mathrm{X}-\mathrm{Y}$ ist in verschiedenen Molekülen und Ionen annähernd konstant und damit charakteristisch, falls sich die Kraftkonstanten nicht zu stark unterscheiden. Für zwei Moleküle a und b mit der gleichen Bindung $\mathrm{X}-\mathrm{Y}$ gilt die bereits von

* Nach Einsetzen der Konstanten erhält man für $298^{\circ} \mathrm{K}$ die Größe $u_{\mathrm{X}-\mathrm{Y}}$ in $\AA$ nach

$$
u \mathrm{X}-\mathrm{Y}=\left[0,004114 f_{\mathrm{X}}-\mathrm{Y}^{-1}-0,01018\left(\mu_{\mathrm{X}}+\mu_{\mathrm{Y}}\right)\right]^{1 / 2},
$$

wenn man $f$ in mdyn $/ \AA$ und $\mu$ in reziproken Atomgewichtseinheiten einsetzt.

4 A. Müller u. S. J. Crvin, J. Mol. Spectr. (im Druck).

5 B. Krebs, A. Müller u. A. F Adini, J. Mol. Spectr. 24, 198 [1967].

\begin{tabular}{|c|c|c|c|c|c|}
\hline \multicolumn{2}{|c|}{ Näherung } & \multirow{2}{*}{$\frac{[4]}{0,044}$} & \multicolumn{2}{|c|}{ Näherung } & \multirow{2}{*}{$\frac{[4]}{0,039}$} \\
\hline $\mathrm{SiO}_{4}^{4-}$ & 0,044 & & $\mathrm{GeF}_{4}$ & 0,038 & \\
\hline $\mathrm{PO}_{4}{ }^{3-}$ & 0,041 & 0,041 & $\mathrm{GeCl}_{4}$ & 0,044 & 0,045 \\
\hline $\mathrm{AsO}_{4}{ }^{3-}$ & 0,040 & 0,041 & $\mathrm{GeBr}_{4}$ & 0,047 & 0,048 \\
\hline $\mathrm{SO}_{4}{ }^{2-}$ & 0,040 & 0,039 & $\mathrm{GeJ}_{4}$ & 0,052 & 0,054 \\
\hline $\mathrm{SeO}_{4}^{2-}$ & 0,039 & 0,039 & $\mathrm{SnCl}_{4}$ & 0,045 & 0,046 \\
\hline $\mathrm{ClO}_{4}^{-}$ & 0,039 & 0,038 & $\mathrm{SnBr}_{4}$ & 0,048 & 0,048 \\
\hline $\mathrm{VO}_{4}{ }^{3-}$ & 0,042 & 0,043 & $\mathrm{SnJ}_{4}$ & 0,054 & 0,055 \\
\hline $\mathrm{CrO}_{4}{ }^{2-}$ & 0,040 & 0,040 & $\mathrm{PbCl}_{4}$ & 0,048 & 0,049 \\
\hline $\mathrm{MoO}_{4}{ }^{2-}$ & 0,039 & 0,039 & $\mathrm{TiCl}_{4}$ & 0,045 & 0,047 \\
\hline $\mathrm{WO}_{4}^{2-}$ & 0,037 & 0,037 & $\mathrm{TiBr}_{4}$ & 0,047 & 0,048 \\
\hline $\mathrm{MnO}_{4}^{-}$ & 0,040 & 0,040 & $\mathrm{ZrCl}_{4}$ & 0,045 & 0,046 \\
\hline $\mathrm{MnO}_{4}{ }^{2-}$ & 0,041 & 0,041 & $\mathrm{VCl}_{4}$ & 0,046 & 0,047 \\
\hline $\mathrm{MnO}_{4}{ }^{3-}$ & 0,042 & 0,043 & $\mathrm{PCl}_{4}^{+}$ & 0,042 & 0,043 \\
\hline $\mathrm{TcO}_{4}^{-}$ & 0,037 & 0,037 & $\mathrm{PBr}_{4}^{+}$ & 0,047 & 0,049 \\
\hline $\mathrm{ReO}_{4}^{-}$ & 0,036 & 0,036 & $\mathrm{AsCl}_{4}^{+}$ & 0,041 & 0,042 \\
\hline $\mathrm{FeO}_{4}{ }^{2-}$ & 0,042 & 0,042 & $\mathrm{SbCl}_{4}^{+}$ & 0,046 & 0,047 \\
\hline $\mathrm{RuO}_{4}$ & 0,037 & 0,037 & ${ }^{11} \mathrm{BF}_{4}^{-}$ & 0,049 & 0,050 \\
\hline $\mathrm{RuO}_{4}^{-}$ & 0,038 & 0,039 & $\mathrm{BCl}_{4}^{-}$ & 0,056 & 0,060 \\
\hline $\mathrm{RuO}_{4}{ }^{2-}$ & 0,039 & 0,039 & $\mathrm{BBr}_{4}-$ & 0,058 & 0,062 \\
\hline $\mathrm{OsO}_{4}$ & 0,035 & 0,035 & $\mathrm{AlCl}_{4}^{-}$ & 0,053 & 0,055 \\
\hline $\mathrm{AsS}_{4}{ }^{3-}$ & 0,048 & 0,049 & $\mathrm{GaCl}_{4}^{-}$ & 0,050 & 0,052 \\
\hline $\mathrm{SbS}_{4}{ }^{3-}$ & 0,049 & 0,050 & $\mathrm{GaBr}_{4}^{-}$ & 0,054 & 0,056 \\
\hline $\mathrm{MoS}_{4}{ }^{2-}$ & 0,041 & 0,043 & $\mathrm{InCl}_{4}^{-}$ & 0,052 & 0,053 \\
\hline $\mathrm{WS}_{4}{ }^{2-}$ & 0,039 & 0,040 & $\mathrm{InBr}_{4}^{-}$ & 0,055 & 0,056 \\
\hline $\mathrm{B}(\mathrm{OH})_{4}^{-}$ & 0,053 & 0,055 & $\mathrm{InJ}_{4}^{-}$ & 0,062 & 0,063 \\
\hline $\mathrm{CF}_{4}$ & 0,046 & 0,044 & $\mathrm{TlBr}_{4}^{-}$ & 0,055 & 0,056 \\
\hline $\mathrm{CCl}_{4}$ & 0,051 & 0,054 & $\mathrm{ZnCl}_{4}{ }^{2-}$ & 0,063 & 0,065 \\
\hline $\mathrm{CBr}_{4}$ & 0,052 & 0,055 & $\mathrm{ZnBr}_{4}{ }^{2-}$ & 0,069 & 0,072 \\
\hline $\mathrm{CJ}_{4}$ & 0,058 & 0,063 & $\mathrm{ZnJ}_{4}{ }^{2-}$ & 0,077 & 0,083 \\
\hline $\mathrm{SiF}_{4}$ & 0,040 & 0,039 & $\mathrm{CdBr}_{4}{ }^{2-}$ & 0,069 & 0,071 \\
\hline $\mathrm{SiCl}_{4}$ & 0,045 & 0,046 & $\mathrm{CdJ}_{4}{ }^{2-}$ & 0,076 & 0,079 \\
\hline $\mathrm{SiBr}_{4}$ & 0,047 & 0,049 & $\mathrm{HgCl}_{4}^{2-}$ & 0,059 & 0,060 \\
\hline $\mathrm{SiJ}_{4}$ & 0,052 & 0,054 & $\mathrm{FeCl}_{4}^{-}$ & 0,053 & 0,054 \\
\hline
\end{tabular}

6 H. Siebert, Anwendungen der Schwingungsspektroskopie in der anorganischen Chemie, Springer, Berlin 1966.
Tab. 1. Mittlere Schwingungsamplituden $u \mathrm{X}-\mathrm{Y}$ in Molekülen

\begin{tabular}{|c|c|c|c|c|c|c|c|}
\hline \multicolumn{3}{|c|}{ Näherung [a] } & {$[\mathrm{b}]$} & \multicolumn{3}{|c|}{ Näherung [a] } & \multirow{2}{*}{$\frac{[\mathrm{b}]}{0,068}$} \\
\hline $\mathrm{NF}_{3}$ & 0,047 & & 0,048 & $\mathrm{GeCl}_{3}-$ & & & \\
\hline $\mathrm{PF}_{3}$ & 0,041 & 0,041 & 0,041 & $\mathrm{SnCl}_{3}^{-}$ & 0,063 & 0,0 & 0,064 \\
\hline $\mathrm{PCl}_{3}$ & 0,049 & & 0,050 & $\mathrm{SnBr}_{3}{ }^{-}$ & & & 0,066 \\
\hline $\mathrm{PBr}_{3}[\mathrm{c}]$ & 0,052 & & 0,051 & $\mathrm{ClO}_{3}^{-}$ & 0,040 & 0,041 & 0,040 \\
\hline $\mathrm{PJ}_{3}[\mathrm{c}]$ & 0,062 & & 0,057 & $\mathrm{BrO}_{3}^{-}$ & 0,039 & 0,0 & 0,040 \\
\hline $\mathrm{AsF}_{3}$ & 0,040 & 0,043 & 0,043 & JO & 0,038 & 0,039 & 0,039 \\
\hline 3 & 0,050 & 0,051 & 0,050 & SO & 0,041 & 0,042 & 0,041 \\
\hline $\mathrm{AsBr}_{3}$ & 0,053 & 0,053 & 0,052 & $\mathrm{SeO}_{3}{ }^{2-}$ & 0,041 & 0,042 & 0,042 \\
\hline $\mathrm{AsJ}_{3}$ & & & 0,058 & $\mathrm{TeO}_{3}{ }^{2-}$ & 0,041 & 0,042 & 0,042 \\
\hline $\mathrm{bCl}_{3}$ & 0,052 & 0,053 & 0,053 & $\mathrm{XeO}_{3}$ & 0,038 & 0,039 & 0,039 \\
\hline 3 & 0,051 & 0,052 & 0,051 & & & & \\
\hline $\mathrm{SeC}$ & 0,047 & 0,048 & 0,048 & & & & \\
\hline $\mathrm{TeCl}_{3}+$ & 0,047 & 0,048 & 0,048 & & & & \\
\hline
\end{tabular}
und Ionen des Typs $\mathrm{XY}_{4}$ mit $\mathrm{T}_{\mathrm{d}}$-Symmetrie (für $298^{\circ} \mathrm{K}$ in $\AA$ ).

a Mit vollständiger Rechnung eines modifizierten Valenzkraftsystems.

b L-Matrix-Näherungsmethode von MüLLER ${ }^{2}$ (vgl. Teil III).

c Mit $f_{\mathrm{P}-\mathrm{Br}}=1,83$ (P.W. Oetuen et al., J. Mol. Spectr. 2, 253 [1958]) und $f_{\mathrm{P}}-\mathrm{J}=1,21 \mathrm{mdyn} / \AA$ (H. Stammreich et al., J. Chem. Phys. 25, 580 [1956]).

Tab. 2. Mittlere Schwingungsamplituden $u_{\mathrm{X}-\mathrm{Y}}$ in Molekülen und Ionen des Typs $\mathrm{XY}_{3}$ mit $\mathrm{C}_{3 \mathrm{v}}$-Symmetrie (für $298^{\circ} \mathrm{K}$ in $\AA$ ). 
MüLLER ${ }^{7,8}$ früher angegebene Näherungsgleichung:

$$
\begin{aligned}
\left.\left(u_{\mathrm{X}-\mathrm{Y}^{\mathrm{a}}}\right)^{2}-\left(u_{\mathrm{X}-\mathrm{Y}}\right)^{\mathrm{b}}\right)^{2} & (4) \\
& =k T\left[\left(f_{\mathrm{X}-\mathrm{Y}^{\mathrm{a}}}\right)^{-1}-\left(f_{\mathrm{X}-\mathrm{Y}^{\mathrm{b}}}\right)^{-1}\right] .
\end{aligned}
$$

2. In isoelektronischen Reihen, wie z. B.

$$
\mathrm{ZnCl}_{4}{ }^{2-}, \mathrm{GaCl}_{4}{ }^{-}, \mathrm{GeCl}_{4}, \mathrm{AsCl}_{4}{ }^{+} \text {, }
$$

nehmen mit zunehmender positiver Gesamtladung die Amplitudenwerte $u_{\mathrm{X}-\mathrm{Y}}$ ab, falls die Kraftkonstanten in dieser Richtung zunehmen, wie es in der Regel der Fall ist. Für isoelektrische Reihen bestimmt in Gl. (3) das erste Glied den Gang der $u$-Werte, da die Atomgewichte in den Horizontalreihen des Periodensystems nur wenig zunehmen.

3. Nach der Abschätzung von $u_{\mathrm{X}-\mathrm{Y}}$ läßt sich für Fälle, in denen lediglich zwei verschiedene Schwingungsamplituden von Bedeutung sind $\left[\mathrm{XY}_{4}\left(\mathrm{~T}_{\mathrm{d}}\right)\right.$, $\left.\mathrm{XY}_{3}\left(\mathrm{D}_{3 \mathrm{~h}}\right), \mathrm{XY}_{3}\left(\mathrm{C}_{3 \mathrm{v}}\right), \mathrm{XY}_{4}\left(\mathrm{D}_{4 \mathrm{~h}}\right), \mathrm{XY}_{6}\left(\mathrm{O}_{\mathrm{h}}\right)\right]$, auch ein Wert für $u_{Y} \ldots \mathrm{Y}$ abschätzen. Für $\mathrm{XY}_{4}\left(\mathrm{~T}_{\mathrm{d}}\right)$ erhält man z. B. folgende Relation (vgl. ${ }^{2}$ ) :

$$
u_{\mathrm{X}-\mathrm{Y}}^{2}=\frac{1}{4}\left[\Sigma_{11}\left(A_{1}\right)+3 \Sigma_{11}\left(F_{2}\right)\right] .
$$

Da sich $\Sigma_{11}\left(A_{1}\right)$ aus $\bar{\nu}_{1}\left(A_{1}\right)$ direkt ergibt, erhält man aus (5) einen Wert für $\Sigma_{11}\left(F_{2}\right)$. Damit läßt sich dann unter Zugrundelegung der beiden Schwingungsfrequenzen in $F_{2}$ die vollständige $\sum$-Matrix in $F_{2}$ und damit auch $u_{\mathrm{Y}} . . \mathrm{Y}$ annähernd berechnen. Aus der unendlichen Lösungsmannigfaltigkeit, die auch bei einem $n=2$-Problem besteht und durch eine
Schwingungsamplitudenellipse repräsentiert wird ${ }^{\mathbf{9}}$, läßt sich hiermit ein vernünftiger Wertebereich abgrenzen.

\section{Normalkoordinatenanalyse}

Die Kraftkonstanten der untersuchten Moleküle und Ionen des Typs $\mathrm{XY}_{3}$ mit $\mathrm{C}_{3 \mathrm{v}}$-Symmetrie wurden durch Lösung der Säkulargleichung

$$
\operatorname{det}|\boldsymbol{G} \boldsymbol{F}-\boldsymbol{\lambda} \boldsymbol{E}|=0
$$

berechnet. Unter Zugrundelegung der Symmetriekoordinaten

$$
\begin{array}{ll}
A_{1}: & S_{1}=3^{-1 / 2} d\left(r_{1}+r_{2}+r_{3}\right), \\
E: \quad & S_{2}=3^{-1 / 2} d\left(\alpha_{1}+\alpha_{2}+\alpha_{3}\right) ; \\
& S_{3 \mathrm{a}}=6^{-1 / 2}\left(2 r_{1}-r_{2}-r_{3}\right), \\
& S_{3 \mathrm{~b}}=2^{-1 / 2}\left(r_{2}-r_{3}\right) \\
& S_{4 \mathrm{a}}=6^{-1 / 2}\left(2 \alpha_{1}-\alpha_{2}-\alpha_{3}\right), \\
& S_{4 \mathrm{~b}}=2^{-1 / 2}\left(\alpha_{2}+\alpha_{3}\right)
\end{array}
$$

ergibt sich die folgende $\boldsymbol{F}$-Matrix eines modifizierten Valenzkraftsystems (M.V.F.F.) :

$$
\begin{array}{lll}
A_{1}: & F_{11}=f_{d}+2 f_{d d}, & F_{22}=f_{a}+2 f_{\alpha a} ; \\
E: & F_{33}=f_{d}-f_{d d}, & F_{44}=f_{a}-f_{a \alpha} .
\end{array}
$$

Die Näherung $F_{12}=F_{34}=0$ ist nur im Falle von $m_{\mathrm{X}}>m_{\mathrm{Y}}$ sinnvoll $^{6}$. Die G-Matrix wurde wie üblich konstruiert und ist der Literatur zu entnehmen. Die der Rechnung zugrunde gelegten Frequenzdaten und

\begin{tabular}{lcccccccccccc}
\hline & $f_{d}$ & $f_{d d}$ & $f_{\alpha}$ & $f_{\alpha \alpha}$ & $L_{11}$ & $L_{12}$ & $L_{21}$ & $L_{22}$ & $L_{33}$ & $L_{34}$ & $L_{43}$ & $L_{44}$ \\
\hline $\mathrm{PF}_{3}$ & 5,09 & 0,56 & 0,63 & 0,18 & 0,2582 & 0,0511 & $-0,2353$ & 0,3542 & 0,3034 & 0,0255 & $-0,2013$ & 0,3837 \\
$\mathrm{AsF}_{3}$ & 3,94 & 0,42 & 0,41 & 0,09 & 0,2452 & 0,0172 & $-0,1023$ & 0,3387 & 0,2619 & 0,0120 & $-0,0928$ & 0,3675 \\
$\mathrm{AsCl}_{3}$ & 2,02 & 0,21 & 0,23 & 0,02 & 0,1925 & 0,0236 & $-0,1476$ & 0,2750 & 0,2081 & 0,0149 & $-0,1045$ & 0,2665 \\
$\mathrm{AsBr}_{3}$ & 1,64 & 0,12 & 0,18 & 0,02 & 0,1451 & 0,0294 & $-0,1888$ & 0,1890 & 0,1662 & 0,0143 & $-0,1225$ & 0,1812 \\
$\mathrm{SbCl}_{3}$ & 1,79 & 0,21 & 0,18 & 0,03 & 0,1831 & 0,0133 & $-0,0899$ & 0,2606 & 0,1941 & 0,0091 & $-0,0706$ & 0,2652 \\
$\mathrm{SbBr}_{3}$ & 1,71 & 0,11 & 0,13 & 0,01 & 0,1362 & 0,0135 & $-0,1225$ & 0,1957 & 0,1472 & 0,0071 & $-0,0794$ & 0,1795 \\
$\mathrm{SeCl}_{3}{ }^{+}$ & 2,24 & 0,15 & 0,33 & 0,05 & 0,1892 & 0,0365 & $-0,1601$ & 0,2628 & 0,2059 & 0,0182 & $-0,1053$ & 0,2642 \\
$\mathrm{TeCl}_{3}{ }^{+}$ & 2,29 & 0,18 & 0,20 & 0,04 & 0,1824 & 0,0138 & $-0,0876$ & 0,2586 & 0,1931 & 0,0068 & $-0,0652$ & 0,2655 \\
$\mathrm{GeCl}_{3}$ & 1,04 & 0,23 & 0,17 & 0,01 & 0,1926 & 0,0285 & $-0,1572$ & 0,2734 & 0,2075 & 0,0307 & $-0,1266$ & 0,2580 \\
$\mathrm{SnCl}_{3}{ }^{-}$ & 1,16 & 0,11 & 0,11 & 0,01 & 0,1909 & 0,0137 & $-0,1084$ & 0,2799 & 0,1913 & 0,0065 & $-0,0526$ & 0,2491 \\
$\mathrm{ClO}_{3}{ }^{-}$ & 5,76 & 0,25 & 1,04 & 0,30 & 0,2632 & 0,0730 & $-0,2172$ & 0,3389 & 0,3121 & 0,0365 & $-0,2045$ & 0,4165 \\
$\mathrm{BrO}_{3}{ }^{-}$ & 5,28 & 0,20 & 0,64 & 0,23 & 0,2578 & 0,0143 & $-0,0625$ & 0,3029 & 0,2811 & 0,0126 & $-0,1029$ & 0,4234 \\
$\mathrm{JO}_{3}{ }^{-}$ & 5,48 & 0,07 & 0,55 & 0,19 & 0,2551 & 0,0068 & $-0,0367$ & 0,2864 & 0,2701 & 0,0067 & $-0,0654$ & 0,4162 \\
$\mathrm{SO}_{3}{ }^{2-}$ & 5,37 & 0,67 & 1,02 & 0,32 & 0,2649 & 0,0697 & $-0,2188$ & 0,3417 & 0,3178 & 0,0449 & $-0,2322$ & 0,4157 \\
$\mathrm{SeO}_{3}{ }^{2-}$ & 4,55 & 0,42 & 0,61 & 0,08 & 0,2652 & 0,0204 & $-0,1025$ & 0,3677 & 0,2777 & 0,0154 & $-0,0853$ & 0,3898 \\
$\mathrm{TeO}_{3}{ }^{2-}$ & 4,36 & 0,31 & 0,49 & 0,07 & 0,2598 & 0,0104 & $-0,0610$ & 0,3523 & 0,2676 & 0,0078 & $-0,0522$ & 0,3853 \\
$\mathrm{XeO}_{3}$ & 5,57 & $-0,11$ & 0,46 & 0,08 & 0,2581 & 0,0076 & $-0,0507$ & 0,3356 & 0,2680 & 0,0050 & $-0,0512$ & 0,3940 \\
\hline
\end{tabular}

Tab. 3. Kraftkonstanten (in mdyn/ $\AA$ ) und L-Matrixelemente [in (a.m.u.) ${ }^{1 / 2}$ ] von Molekülen und Ionen des Typs $\mathrm{XY}_{3}$ mit $\mathrm{C}_{3 \mathrm{v}}$-Symmetrie.

7 A. Müller, Naturwiss. 53, 701 [1966].

9 S. J. Cyvin, J. Mol. Spectr. 6, 338 [1961].

8 A. Müller, Z. Phys. Chem. 236, 305 [1967]. 


\begin{tabular}{|c|c|c|c|c|c|c|c|c|c|c|c|}
\hline & & $f_{d}$ & $f_{d d}$ & $f_{\alpha}$ & $f_{\alpha \alpha}$ & & & $f_{d}$ & $f_{d d}$ & $f_{\alpha}$ & $f_{\alpha \alpha}$ \\
\hline $\mathrm{PF}_{3}$ & $\begin{array}{l}1 \\
2 \\
3 \\
4\end{array}$ & $\begin{array}{l}0,72 \\
0,09 \\
1,07 \\
0,05\end{array}$ & $\begin{array}{r}0,16 \\
0,02 \\
-0,12 \\
-0,01\end{array}$ & $\begin{array}{l}0,08 \\
0,57 \\
0,06 \\
1,33\end{array}$ & $\begin{array}{r}0,04 \\
0,32 \\
-0,01 \\
-0,37\end{array}$ & $\mathrm{GeCl}_{3}^{-}$ & $\begin{array}{l}1 \\
2 \\
3 \\
4\end{array}$ & $\begin{array}{l}0,64 \\
0,05 \\
1,19 \\
0,09\end{array}$ & $\begin{array}{r}0,28 \\
0,02 \\
-0,26 \\
-0,02\end{array}$ & $\begin{array}{l}0,07 \\
0,82 \\
0,07 \\
1,00\end{array}$ & $\begin{array}{r}0,01 \\
0,11 \\
0,00 \\
-0,07\end{array}$ \\
\hline $\mathrm{AsF}_{3}$ & $\begin{array}{l}1 \\
2 \\
3 \\
4\end{array}$ & $\begin{array}{l}0,81 \\
0,02 \\
1,10 \\
0,01\end{array}$ & $\begin{array}{r}0,17 \\
0,00 \\
-0,12 \\
0,00\end{array}$ & $\begin{array}{l}0,01 \\
0,69 \\
0,02 \\
1,25\end{array}$ & $\begin{array}{r}0,01 \\
0,29 \\
0,00 \\
-0,26\end{array}$ & $\mathrm{SnCl}_{3}^{-}$ & $\begin{array}{l}1 \\
2 \\
3 \\
4\end{array}$ & $\begin{array}{l}0,82 \\
0,02 \\
1,10 \\
0,01\end{array}$ & $\begin{array}{r}0,16 \\
0,00 \\
-0,11 \\
0,00\end{array}$ & $\begin{array}{l}0,02 \\
0,87 \\
0,01 \\
1,06\end{array}$ & $\begin{array}{r}0,00 \\
0,11 \\
0,00 \\
-0,07\end{array}$ \\
\hline $\mathrm{AsCl}_{3}$ & $\begin{array}{l}1 \\
2 \\
3 \\
4\end{array}$ & $\begin{array}{l}0,78 \\
0,05 \\
1,08 \\
0,03\end{array}$ & $\begin{array}{r}0,16 \\
0,01 \\
-0,11 \\
0,00\end{array}$ & $\begin{array}{l}0,05 \\
0,77 \\
0,03 \\
1,09\end{array}$ & $\begin{array}{r}0,01 \\
0,17 \\
0,00 \\
-0,12\end{array}$ & $\mathrm{ClO}_{3}^{-}$ & $\begin{array}{l}1 \\
2 \\
3 \\
4\end{array}$ & $\begin{array}{l}0,78 \\
0,14 \\
0,99 \\
0,06\end{array}$ & $\begin{array}{r}0,07 \\
0,01 \\
-0,04 \\
0,00\end{array}$ & $\begin{array}{l}0,10 \\
0,54 \\
0,07 \\
1,32\end{array}$ & $\begin{array}{r}0,05 \\
0,31 \\
-0,02 \\
-0,38\end{array}$ \\
\hline $\mathrm{AsBr}_{3}$ & $\begin{array}{l}1 \\
2 \\
3 \\
4\end{array}$ & $\begin{array}{l}0,73 \\
0,15 \\
1,02 \\
0,06\end{array}$ & $\begin{array}{r}0,10 \\
0,02 \\
-0,07 \\
0,00\end{array}$ & $\begin{array}{l}0,14 \\
0,68 \\
0,06 \\
1,06\end{array}$ & $\begin{array}{r}0,03 \\
0,15 \\
-0,01 \\
-0,12\end{array}$ & $\mathrm{BrO}_{3}^{-}$ & $\begin{array}{l}1 \\
2 \\
3 \\
4\end{array}$ & $\begin{array}{l}0,92 \\
0,01 \\
1,03 \\
0,01\end{array}$ & $\begin{array}{r}0,07 \\
0,00 \\
-0,04 \\
0,00\end{array}$ & $\begin{array}{l}0,01 \\
0,57 \\
0,02 \\
1,55\end{array}$ & $\begin{array}{r}0,00 \\
0,42 \\
-0,01 \\
-0,56\end{array}$ \\
\hline $\mathrm{SbCl}_{3}$ & $\begin{array}{l}1 \\
2 \\
3 \\
4\end{array}$ & $\begin{array}{l}0,79 \\
0,02 \\
1,12 \\
0,01\end{array}$ & $\begin{array}{r}0,19 \\
0,00 \\
-0,13 \\
0,00\end{array}$ & $\begin{array}{l}0,02 \\
0,75 \\
0,01 \\
1,17\end{array}$ & $\begin{array}{r}0,00 \\
0,23 \\
0,00 \\
-0,18\end{array}$ & $\mathrm{JO}_{3}^{-}$ & $\begin{array}{l}1 \\
2 \\
3 \\
4\end{array}$ & $\begin{array}{l}0,97 \\
0,00 \\
1,01 \\
0,00\end{array}$ & $\begin{array}{r}0,03 \\
0,00 \\
-0,01 \\
0,00\end{array}$ & $\begin{array}{l}0,00 \\
0,59 \\
0,00 \\
1,52\end{array}$ & $\begin{array}{r}0,00 \\
0,41 \\
0,00 \\
-0,52\end{array}$ \\
\hline $\mathrm{SbBr}_{3}$ & $\begin{array}{l}1 \\
2 \\
3 \\
4\end{array}$ & $\begin{array}{l}0,83 \\
0,05 \\
1,05 \\
0,02\end{array}$ & $\begin{array}{r}0,11 \\
0,01 \\
-0,07 \\
0,00\end{array}$ & $\begin{array}{l}0,05 \\
0,82 \\
0,02 \\
1,06\end{array}$ & $\begin{array}{r}0,01 \\
0,13 \\
0,00 \\
-0,08\end{array}$ & $\mathrm{SO}_{3}{ }^{2-}$ & $\begin{array}{l}1 \\
2 \\
3 \\
4\end{array}$ & $\begin{array}{l}0,69 \\
0,12 \\
1,06 \\
0,08\end{array}$ & $\begin{array}{r}0,17 \\
0,03 \\
-0,13 \\
-0,01\end{array}$ & $\begin{array}{l}0,09 \\
0,52 \\
0,10 \\
1,36\end{array}$ & $\begin{array}{r}0,05 \\
0,33 \\
-0,03 \\
-0,43\end{array}$ \\
\hline $\mathrm{SeCl}_{3}+$ & $\begin{array}{l}1 \\
2 \\
3 \\
4\end{array}$ & $\begin{array}{l}0,79 \\
0,09 \\
1,04 \\
0,04\end{array}$ & $\begin{array}{r}0,11 \\
0,01 \\
-0,07 \\
0,00\end{array}$ & $\begin{array}{l}0,08 \\
0,70 \\
0,04 \\
1,12\end{array}$ & $\begin{array}{r}0,02 \\
0,20 \\
-0,01 \\
-0,16\end{array}$ & $\mathrm{SeO}_{3}{ }^{2-}$ & $\begin{array}{l}1 \\
2 \\
3 \\
4\end{array}$ & $\begin{array}{l}0,83 \\
0,02 \\
1,09 \\
0,01\end{array}$ & $\begin{array}{r}0,15 \\
0,00 \\
-0,10 \\
0,00\end{array}$ & $\begin{array}{l}0,02 \\
0,78 \\
0,01 \\
1,14\end{array}$ & $\begin{array}{r}0,00 \\
0,20 \\
0,00 \\
-0,15\end{array}$ \\
\hline \multirow[t]{2}{*}{$\mathrm{TeCl}_{3}{ }^{+}$} & $\begin{array}{l}1 \\
2 \\
3 \\
4\end{array}$ & $\begin{array}{l}0,84 \\
0,02 \\
1,07 \\
0,01\end{array}$ & $\begin{array}{r}0,13 \\
0,00 \\
-0,08 \\
0,00\end{array}$ & $\begin{array}{l}0,02 \\
0,68 \\
0,01 \\
1,27\end{array}$ & $\begin{array}{r}0,01 \\
0,30 \\
0,00 \\
-0,28\end{array}$ & $\mathrm{TeO}_{3}{ }^{2-}$ & $\begin{array}{l}1 \\
2 \\
3 \\
4\end{array}$ & $\begin{array}{l}0,87 \\
0,01 \\
1,07 \\
0,00\end{array}$ & $\begin{array}{r}0,12 \\
0,00 \\
-0,08 \\
0,00\end{array}$ & $\begin{array}{l}0,01 \\
0,78 \\
0,01 \\
1,16\end{array}$ & $\begin{array}{r}0,00 \\
0,21 \\
0,00 \\
-0,16\end{array}$ \\
\hline & & & & & & $\mathrm{XeO}_{3}$ & $\begin{array}{l}1 \\
2 \\
3 \\
4\end{array}$ & $\begin{array}{l}1,04 \\
0,01 \\
0,98 \\
0,00\end{array}$ & $\begin{array}{r}-0,04 \\
0,00 \\
0,02 \\
0,00\end{array}$ & $\begin{array}{l}0,00 \\
0,74 \\
0,00 \\
1,20\end{array}$ & $\begin{array}{r}0,00 \\
0,25 \\
0,00 \\
-0,20\end{array}$ \\
\hline
\end{tabular}

Tab. 4. Potentialenergieverteilung.

Bindungswinkel sind dem Buch von Siebert ${ }^{6}$ entnommen. Für die dort nicht aufgeführten Bindungswinkel wurden folgende Werte angenommen: $\mathrm{PBr}_{3}$ und $\mathrm{PJ}_{3}: 100^{\circ} ; \mathrm{AsBr}_{3}: 98,5^{\circ} ; \mathrm{AsJ}_{3}: 100,2^{\circ}$; $\mathrm{SbBr}_{3}: 97^{\circ}$ und $\mathrm{SnBr}_{3}: 95^{\circ}$ (vgl. ${ }^{10}$ ), sowie $102,1^{\circ}$ für $\mathrm{NF}_{3}$. Die berechneten Kraftkonstanten und $\mathbf{L}$ Matrizen sind in Tab. 3 angegeben. Die Potentialenergieverteilungen sind Tab. $4 \mathrm{zu}$ entnehmen. Es wurden nur für diejenigen Moleküle und Ionen Rechnungen durchgeführt, für die das M.V.F.F. vernünftig ist $\left(\mathrm{vgl} .{ }^{6}\right)$.

10 L. E. Sutron (Ed.), Tables of Interatomic Distances and Configuration in Molecules and Ions, The Chemical Society, London 1958.

\section{Berechnung von mittleren Schwingungs- amplituden nach der L-Matrix- Näherungsmethode}

In einer früheren Arbeit wurde von MüLLER 2 eine neue einfache Methode zur Näherungsberechnung von mittleren Schwingungsamplituden angegeben, die hier L-Matrix-Näherungsmethode genannt werden soll. Die Methode gestattet es, bei Säkulargleichungsproblemen der Ordnung $n=2$, bei denen eine Valenz- und eine Deformationsschwingung miteinander gekoppelt sind, ohne Lösung eines Eigenwertproblems zuverlässige $u$-Werte zu berechnen.

Aus

$$
\boldsymbol{G}=\mathbf{L} \mathbf{L}^{\prime}
$$

folgt für $n=2$ 


$$
\begin{aligned}
& G_{11}=L_{11}{ }^{2}+L_{12}{ }^{2}, \\
& G_{22}=L_{21}{ }^{2}+L_{22}{ }^{2}, \\
& G_{12}=L_{11} L_{21}+L_{12} L_{22} .
\end{aligned}
$$

Mit der Näherung

$$
L_{12}=0
$$

( $v_{1}$ : Valenzschwingung, $v_{2}$ : Deformationsschwingung) kann man aus (7) eine angenäherte L-Matrix berechnen (vgl. ${ }^{2}$ ). Für zahlreiche Moleküle des Typs $\mathrm{XY}_{4}\left(\mathrm{~T}_{\mathrm{d}}\right)$ und $\mathrm{XY}_{3}\left(\mathrm{D}_{3 \mathrm{~h}}\right)$ stimmen die mit der L-Matrix-Näherungsmethode berechneten $u$-Werte ausgezeichnet mit denen aus Elektronenbeugungsuntersuchungen oder mit Werten aus vollständigen Rechnungen überein ${ }^{2}$.

Aus der $\mathbf{L}$-Matrix lassen sich dann nach der bekannten Gleichung

$$
\boldsymbol{\Sigma}=\mathbf{L} \boldsymbol{\Delta} \boldsymbol{L}^{\prime}
$$

mittlere Schwingungsamplituden berechnen. Im vorliegenden Fall $\mathrm{XY}_{3}\left(\mathrm{C}_{3 \mathrm{v}}\right)$ erhält man folgende Gleichungen:

$$
\begin{aligned}
u_{\mathrm{X}-\mathrm{Y}^{2}} & =\frac{1}{3} \Sigma_{11}+{ }_{3}^{2} \Sigma_{33}, \\
u_{\mathrm{Y} \ldots \mathrm{Y}^{2}} & =\frac{2}{3}\left(2 \Sigma_{11}+\Sigma_{33}\right) \sin ^{2}(\Theta / 2) \\
& +\frac{1}{3}\left(\Sigma_{22}+2 \Sigma_{44}\right) \cos ^{2}(\Theta / 2) \\
& +\frac{4}{3}\left(\Sigma_{12}+\Sigma_{34}\right) \sin (\Theta / 2) \cos (\Theta / 2),
\end{aligned}
$$

$(\Theta$ : Bindungswinkel). Mit (8), (9), (10) und (11) ergeben sich somit die aus Tab. 2 (Spalte 4) und
Tab. $5 \mathrm{zu}$ entnehmenden mittleren Schwingungsamplituden. Zum Vergleich sind die Amplitudenwerte angegeben, die mit den aus Tab. 4 zu entnehmenden $\mathbf{L}$-Matrizen berechnet wurden. Die Übereinstimmung ist auch hier ausgezeichnet. Die bisher in der Literatur angegebenen Werte für die hier untersuchten Moleküle und Ionen sind zum größten Teil falsch (vgl. hierzu ${ }^{1}$ ).

\begin{tabular}{lcclcc}
\hline & {$[a]$} & $\begin{array}{c}\text { L-Matrix- } \\
\text { Näherung }\end{array}$ & [a] & $\begin{array}{c}\text { L-Matrix- } \\
\text { Näherung }\end{array}$ \\
\hline $\mathrm{NF}_{3}$ & & 0,056 & $\mathrm{TeCl}_{3}{ }^{+}$ & 0,109 & 0,106 \\
$\mathrm{PF}_{3}$ & 0,071 & 0,068 & $\mathrm{GeCl}_{3}{ }^{-}$ & 0,123 & 0,116 \\
$\mathrm{PC}_{3}$ & & 0,086 & $\mathrm{SnCl}_{3}{ }^{-}$ & 0,153 & 0,149 \\
$\mathrm{PBr}_{3}$ & & 0,093 & $\mathrm{SnBr}_{3}{ }^{-}$ & & 0,161 \\
$\mathrm{PJ}_{3}$ & & 0,110 & $\mathrm{ClO}_{3}{ }^{-}$ & 0,063 & 0,060 \\
$\mathrm{AsF}_{3}$ & 0,082 & 0,080 & $\mathrm{BrO}_{3}{ }^{-}$ & 0,072 & 0,070 \\
$\mathrm{AsCl}_{3}$ & 0,104 & 0,100 & $\mathrm{JO}_{3}{ }^{-}$ & 0,074 & 0,073 \\
$\mathrm{AsBr}_{3}$ & 0,113 & 0,107 & $\mathrm{SO}_{3}{ }^{-}$ & 0,064 & 0,061 \\
$\mathrm{AsJ}_{3}{ }^{-}$ & & 0,121 & $\mathrm{SeO}_{3}{ }^{2-}$ & 0,073 & 0,072 \\
$\mathrm{SbCl}_{3}{ }^{+}$ & 0,116 & 0,112 & $\mathrm{TeO}_{3}{ }^{2-}$ & 0,079 & 0,077 \\
$\mathrm{SbBr}_{3}{ }^{+}$ & 0,131 & 0,127 & $\mathrm{XeO}_{3}$ & 0,078 & 0,078 \\
$\mathrm{SeCl}_{3}{ }^{+}$ & 0,091 & 0,087 & & & \\
\hline
\end{tabular}

[a] Mit vollständiger Rechnung eines modifizierten Valenzkraftsystems.

Tab.5. Mit Hilfe der L-Matrix-Näherungsmethode ${ }^{2}$ ermittelte mittlere Schwingungsamplituden $u \mathrm{Y} \ldots \mathrm{Y}$ in Molekülen und Ionen des Typs $\mathrm{XY}_{3}$ mit $\mathrm{C}_{3 \mathrm{v}}$-Symmetrie (Werte für gebundene Abstände vgl. Tab. 2). Die Werte (in $\AA$ ) gelten für $298^{\circ} \mathrm{K}$.

Wir danken Herrn Prof. Dr. O. Glemser sehr für großzügige Unterstützung. 